\title{
Estudio comparativo entre enoxaparina y ácido acetilsalicílico en profilaxis antitrombótica para pacientes sometidos a artroplastía total de rodilla
}

\author{
Comparative study between enoxaparin and salicylic acetyl acid in antithrombotic \\ prophylaxis for patients undergoing total knee arthroplasty \\ Cortes-De la Fuente AA, * Villalobos-Campuzano C, \\ Bucio-Paticio B, ${ }^{\ddagger}$ Valencia-Martínez G, ${ }^{\S}$ Martínez-Montiel O»
}

ISSEMyM Toluca.

RESUMEN. Introducción: Aún existe controversia en cuanto a la tromboprofilaxis para la disminución de la enfermedad tromboembólica en la cirugía ortopédica mayor. Objetivo: Responder la siguiente pregunta: ¿existe diferencia en la efectividad y seguridad en el manejo antitrombótico de pacientes con un régimen tradicional de enoxaparina contra ácido acetilsalicílico? Material y métodos: Las cirugías se llevaron a cabo por tres cirujanos, se aleatorizó la muestra y los pacientes fueron sometidos a los criterios del estudio. Evaluamos eficacia y seguridad así como la necesidad de reingreso y variables secundarias como infección, infarto agudo de miocardio, enfermedad vascular cerebral y muerte con un seguimiento de 90 días. Resultados: El total de la muestra fue de 402 pacientes, 214 en el grupo de enoxaparina y 188 en el de aspirina. Se presentaron cinco casos $(1.24 \%)$ con enfermedad tromboembólica, tres $(1.4 \%)$ enoxaparina y dos $(1.06 \%)$ aspirina sin diferencia significativa $(p=0.23)$. En cuanto a seguridad, el sangrado mayor
ABSTRACT. Introduction: There is still controversy regarding thrombo-prophylaxis for the reduction of thromboembolic disease in major orthopedic surgery. Objective: To answer the following question: is there a difference in the effectiveness and safety in the antithrombotic management of patients with a traditional regimen of enoxaparin against acetyl salicylic acid? Material and methods: The surgeries were performed by 3 surgeons; the sample was randomized and the patients were subjected to the study criteria. We evaluated efficacy and safety as well as the need for readmission and secondary variables such as infection, acute myocardial infarction (AMI), cerebral vascular disease and death with a followup of 90 days. Results: The total sample was 402 patients; 214 in the enoxaparin group and 188 in the aspirin group. There were 5 cases $(1.24 \%)$ with thromboembolic disease, 3 $(1.4 \%)$ enoxaparin and $2(1.06 \%)$ aspirin without significant difference $(p=0.23)$. In terms of safety, major bleeding

\section{Nivel de evidencia: II, Estudio prospectivo comparativo.}

* Residente de Traumatología y Ortopedia.

${ }^{\ddagger}$ MB de Traumatología y Ortopedia.

§ Jefe de servicio y profesor principal del programa de Subespecialidad de Cirugía articular, Ortopedia en CM ISSEMyM Toluca.

" MB de Traumatología y Ortopedia y profesor adjunto del programa de Subespecialidad de Cirugía articular.

Correspondencia:

Dr. Adrián Aziz Cortes-De la Fuente

Esteban Plata Núm. 217, Morelos Segunda Secc, C.P. 50120,

Toluca de Lerdo, Estado de México.

E-mail: adrianazizcortesdelafuente@gmail.com

Recibido: 22-04-2020. Aceptado: 25-01-2021.

Citar como: Cortes-De la Fuente AA, Villalobos-Campuzano C, Bucio-Paticio B, Valencia-Martínez G, Martínez-Montiel O. Estudio comparativo entre enoxaparina y ácido acetilsalicílico en profilaxis antitrombótica para pacientes sometidos a artroplastía total de rodilla. Acta Ortop Mex. 2021; 35(2): 163-168. https://dx.doi.org/10.35366/101860

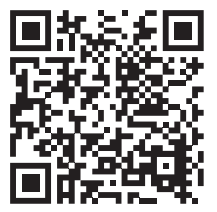


fue cero en ambos grupos, presentándose sangrado menor en siete pacientes (1.74\%), cuatro (1.86\%) fueron del grupo enoxaparina y tres $(1.59 \%)$ del grupo aspirina sin diferencias significativas $(\mathrm{p}=0.82)$. Los resultados secundarios mostraron cinco (1.24\%) infecciones de herida quirúrgica superficiales y un IAM en los primeros 30 días del procedimiento en el grupo de enoxaparina. Conclusión: La aspirina como monoterapia es segura y eficaz en profilaxis antitrombótica en pacientes operados de artroplastía total de rodilla.

Palabras clave: Profilaxis, antitrombótica, ácido acetilsalicílico, cirugía, prótesis, rodilla.

\section{Introducción}

La enfermedad tromboembólica engloba la trombosis venosa profunda (TVP) y tromboembolia pulmonar (TEP), lo que implica un riesgo en cirugía mayor ortopédica como la artroplastía total de rodilla presentándose en $1.08 \%$. Sin tromboprofilaxis, la trombosis venosa profunda puede ocurrir hasta en $35 \%$, se presenta en $94 \%$ las primeras dos semanas y en $89 \%$ en la primera semana. La tromboembolia pulmonar es responsable de entre 11.7 y $17.1 \%$ de la mortalidad en los primeros 90 días del postquirúrgico, del infarto agudo de miocardio (IAM) en $25.9 \%$, de la enfermedad vascular cerebral (EVC) en $0.2 \% .^{1,2,3}$

El empleo de aspirina como terapia antitrombótica de elección ha aumentado por su cómoda administración, por no requerir monitorización, por asociarse con un bajo índice de sangrado y una eficacia adecuada nivel $1 \mathrm{~B}$ con reducciones de 36\% de TVP sintomática. ${ }^{4,5}$ La dosis recomendada por la Academia Americana de Ortopedia es de 325 mg/día recomendación $1 \mathrm{C}$, por lo que los estudios son insuficientes, otras guías internacionales que apoyan su uso encuentran discrepancias significativas sobre el momento ideal para el inicio de la terapia, dosis, duración y las posibles modificaciones con respecto a pacientes ya anticoagulados o con factores de riesgo bien definidos. ${ }^{1,6,7}$ Se ha documentado que dosis de entre 30 y $150 \mathrm{mg}$ son suficientes para inhibir la COX-1.

Los estudios apoyan el empleo de aspirina por el bajo costo, la seguridad histórica, la efectividad y la conveniencia como una opción distinta a heparinas de bajo peso molecular. Jameson y colaboradores reportaron el costo anual de la tromboprofilaxis en el Reino Unido y Gales con el empleo de agentes potentes tras artroplastía total de rodilla (ATR) y artroplastía total de cadera (ATC) siendo de 13,000,000 de libras contra 110,000 si se utilizara aspirina. ${ }^{8,9,10}$

Se ha visto relacionada con menor drenaje persistente por la herida, menor formación de hematomas e infecciones, prevención de TVP proximal y distal y disminución de TEP fatal y no fatal. ${ }^{11,12}$

Muchos estudios han demostrado aumento de riesgo de sangrado mayor o menor y complicaciones de herida con was zero in both groups, with minor bleeding in 7 patients (1.74\%), 4 (1.86\%) were from the enoxaparin group and $3(1.59 \%)$ from the aspirin group without significant differences $(p=0.82)$. Secondary outcomes showed 5 $(1.24 \%)$ superficial surgical wound infections and one AMI in the first 30 days of the procedure in the enoxaparin group. Conclusion: Aspirin as monotherapy is safe, effective in antithrombotic prophylaxis in patients operated on total knee arthroplasty.

Keywords: Prophylaxis, antithrombotic, acetylsalicylic acid, surgery, prosthesis, knee.

aplicación de heparina. ${ }^{13}$ Además, han manifestado que la aplicación de otros agentes, como rivaroxaban, causan mayor sangrado oculto y complicaciones.

Presenta ventajas sin intención reportadas en múltiples estudios como la disminución del índice de mortalidad a los 45 días y a largo plazo, reducción en la cantidad de analgésicos y en especial, de opioides en el postquirúrgico. ${ }^{14}$

Se puede administrar sin problema con un inhibidor de la COX-II. Existen algoritmos publicados para su utilización en pacientes con riesgo elevado en los que se recomendaría el uso de heparina vs. aspirina:

- Pacientes con ciertas condiciones como hipercoagulabilidad, cáncer metastásico, etc., sepsis, enfermedad pulmonar obstructiva crónica (EPOC), las cuales colocan al paciente en un riesgo incrementado de tromboembolia de más de 3\%. ${ }^{1,11}$

Por lo anterior, se debería considerar al ácido acetilsalicílico como una opción de esquema unificado para nuestro país por su bajo costo, facilidad para su adquisición, mayor apego, alta efectividad y seguridad probada.

La trombosis es responsable de una cuarta parte de las muertes a nivel mundial. La enfermedad tromboembólica en países con bajos, medianos y altos ingresos es la causa principal de muerte asociada a la hospitalización. ${ }^{15,16}$ Como se ha tratado en el apartado previo, las personas que son sometidas a una cirugía ortopédica mayor de artroplastía de rodilla o cadera presentan mayor riesgo de enfermedad tromboembólica venosa (ETV), $1.08 \%$ en el caso de reemplazo de rodilla, por lo que se debe utilizar una profilaxis farmacológica y mecánica además de la deambulación temprana en el postoperatorio. ${ }^{17}$

En México se desconoce estadística alguna sobre la tromboprofilaxis administrada de primera línea y elección para los ortopedistas que realizan reemplazos articulares; no existe registro de la cifra exacta de eventos de enfermedad tromboembólica en nuestra población.

La ETV se presenta en más de $25 \%$ de los pacientes quirúrgicos hospitalizados. Los esquemas empleados varían entre 34 y 58\% con los siguientes argumentos: riesgo de 
hemorragia, exclusión de ciertos tipos de pacientes y desconocimiento de la ETV. ${ }^{18}$

Basados en cifras de otros países, los eventos tromboembólicos venosos son un problema de salud significativo y una preocupación importante tras la cirugía ortopédica mayor, ya que son responsables de muerte directa en una de cada cuatro artroplastías de cadera y, sin conocer el dato exacto en cirugía de rodilla, se estima algo similar.

En una economía creciente como la de México las opciones con que se cuenta para realizar una adecuada prevención deben ser difundidas por los profesionales de la salud para su utilización en medio privado y específicamente, para el ahorro en el sector público.

Se deben realizar estudios comparativos en nuestras poblaciones para sustentar la utilización de agentes para tromboprofilaxis más económica, cómoda, segura y efectiva para los pacientes.

Existen guías internacionales que ya apoyan el uso de algunos agentes; sin embargo, hay discrepancias significativas sobre qué terapia es la más adecuada, el uso de aspirina como profilaxis, el momento ideal del inicio de las terapias farmacológicas y su duración, así como las modificaciones con respecto a pacientes ya anticoagulados o con factores de riesgo bien definidos.

La responsabilidad es compartida entre instituciones gubernamentales y médicos en proporcionar medidas para la experimentación controlada con terapias seguras para mitigar el costo excesivo en medicamentos y disminuir complicaciones que llevan a costos más elevados por readmisiones.

Es de suma importancia realizar un consenso basado en evidencias sobre la administración de estos agentes para estandarizar medidas de profilaxis trombótica. Por lo tanto, la pregunta de investigación es: ¿existe diferencia en la efectividad y seguridad en el manejo antitrombótico de pacientes con un régimen tradicional de enoxaparina postquirúrgica contra ácido acetilsalicílico?

\section{Material y métodos}

Realizamos un ensayo clínico controlado para valorar si existe diferencia en la efectividad y seguridad en el manejo antitrombótico de pacientes con un régimen tradicional de enoxaparina postquirúrgica contra ácido acetilsalicílico, una vez que se ha realizado el diagnóstico, los pacientes fueron sometidos a los criterios de selección.

Como inclusión se consideran candidatos a reemplazo articular pacientes de 18 a 90 años y de sexo indistinto y excluimos las artroplastías bilaterales en pacientes con alguna contraindicación para alguno de los esquemas terapéuticos, quienes toman anticoagulación de más de seis meses diferente a antiplaquetarios, pacientes con antecedentes de sangrado gastrointestinal, cerebral u otra hemorragia documentada, enfermedades en tratamientos alternativos que afecten su hemostasia, con descontrol hipertensivo de $>220 / 120$, alergias conocidas a ácido acetilsalicílico y/o heparina y pacientes con cáncer metastásico.

\begin{tabular}{|c|c|c|c|}
\hline & $\begin{array}{c}\text { Muestra } \\
\mathrm{N}=402, \mathrm{n}(\%)\end{array}$ & $\begin{array}{c}\text { Enoxaparina } \\
\mathrm{N}=214, \mathrm{n}(\%)\end{array}$ & $\begin{array}{c}\text { Aspirina } \\
\mathrm{N}=188, \mathrm{n}(\%)\end{array}$ \\
\hline \multicolumn{4}{|l|}{ Resultados demográficos e información quirúrgica } \\
\hline Edad, media & 70.85 & 71.31 & 70.33 \\
\hline Hombres & (51) & 106 (49) & $101(54)$ \\
\hline Mujeres & (49) & $108(51)$ & $87(46)$ \\
\hline \multicolumn{4}{|l|}{ Antecedentes de importancia } \\
\hline Diabetes & 167 & 92 & 75 \\
\hline IR (insuficiencia renal) & 4 & 4 & 0 \\
\hline TEP o TVP previa & 0 & 0 & 0 \\
\hline HAS & 158 & 69 & 89 \\
\hline Hiperlipidemia & 43 & 23 & 20 \\
\hline Cirugía en los últimos seis meses & 12 & 4 & 8 \\
\hline Angina & 10 & 6 & 4 \\
\hline IAM previo & 14 & 7 & 7 \\
\hline Fumador & 98 & 47 & 51 \\
\hline \multicolumn{4}{|l|}{ ASA } \\
\hline I & $97(24)$ & $47(49)$ & $50(51)$ \\
\hline II & 277 (69) & $132(48)$ & $145(52)$ \\
\hline III & $24(6)$ & $11(46)$ & $13(54)$ \\
\hline IV & $4(1)$ & $4(100)$ & 0 \\
\hline \multicolumn{4}{|l|}{ Cirugía realizada } \\
\hline ATR derecha & & 102 & 119 \\
\hline ATR izquierda & & 112 & 69 \\
\hline Tiempo de estancia hospitalaria & & 3.2 & 3.6 \\
\hline Promedio de pérdida sanguínea durante procedimiento $\left(\mathrm{cm}^{3}\right)$ & & 468 & 428 \\
\hline
\end{tabular}




\begin{tabular}{|c|c|c|c|c|}
\hline \multirow[b]{2}{*}{ Resultados } & \multirow[b]{2}{*}{ n (\%) } & \multicolumn{2}{|c|}{ Eficacia } & \multirow[b]{2}{*}{$\mathrm{p}$} \\
\hline & & $\begin{array}{c}\text { Enoxaparina } \\
\mathrm{N}=214 \\
\mathrm{n}(\%)\end{array}$ & $\begin{array}{c}\text { Aspirina } \\
\text { N =188 } \\
\text { n (\%) }\end{array}$ & \\
\hline Total & $5(1.24)$ & $3(1.4)$ & $2(1.06)$ & 0.232390 \\
\hline $\begin{array}{l}\text { Embolismo } \\
\text { pulmonar }\end{array}$ & $3(0.74)$ & $2(0.93)$ & $1(0.53)$ & \\
\hline $\begin{array}{l}\text { Trombosis } \\
\text { venosa profunda } \\
\text { sintomática }\end{array}$ & $3(0.49)$ & $1(0.46)$ & $1(0.53)$ & \\
\hline
\end{tabular}

Posteriormente fueron aleatorizados mediante la plataforma de Research Randomizer Form v4.0, con lo que determinamos el tipo de tratamiento que se le otorgará al paciente y su esquema de tromboprofilaxis.

Se evaluaron ambos esquemas como resultado principal de efectividad definida por el número de readmisiones hospitalarias, enfermedad tromboembólica, evento separado de trombosis venosa profunda sintomática y tromboembolia pulmonar.

Seguridad como sangrado mayor: sangrado fatal, sintomático en un área u órgano crítico o sangrado que causó una disminución de $20 \mathrm{~g} / 1$ o más en el nivel de hemoglobina o resultado en la transfusión de dos o más unidades de sangre y sangrado menor como relevante, pero sin dar lugar a hospitalización, aspiración de hematoma de la herida, hematoma con sangrado manifiesto como gastrointestinal, epistaxis, hematuria, equimosis, hemoptisis y/o hemorragia conjuntival.

Al total de la muestra se le sometió a un seguimiento a los 15, 30 y 90 días del postquirúrgico analizando variables mediante $t$ de Student para probar una hipótesis nula con diferencia para variables desapareadas de seguridad y eficacia como resultados primarios e infección, IAM, EVC y muerte como resultados secundarios. El análisis de costo univariable se redujo al promedio por tratamiento por paciente y por grupo experimental.

La cirugía se llevó a cabo por un grupo de cirujanos experimentados en reemplazo articular, todos utilizaron isquemia en el procedimiento, la aplicación de la tromboprofilaxis fue realizada por el médico a cargo del estudio:

Grupo experimental 1: aspirina con capa entérica. Aspirina, dos tabletas de $100 \mathrm{mg}$ el día de la cirugía. Comenzar el día uno postoperatorio con la dosis de 100 mg hasta completar 30 días.

Grupo experimental 2: enoxaparina SC. Enoxaparina 40-60 mg c/24 mg comenzando el día uno postoperatorio y por 30 días después del egreso comenzando a las 12 horas del postquirúrgico por 30 días.

Cada paciente fue informado del tipo de estudio que se está realizando así como de la posibilidad de aplicación de cualquiera de los tratamientos. Se le explican los riesgos y los beneficios y se le entrega un consentimiento informado, el cual fue elaborado de acuerdo con los lineamientos de la declaración de Helsinki y la Ley General de Salud mexicana en los artículos 20, 21 y 22. La identidad de los pacientes es estrictamente confidencial. El protocolo se realizó con previa autorización de comité de ética del hospital.

\section{Resultados}

Al inicio del estudio se instituyó una muestra de 430 pacientes para conformar dos poblaciones de 215 pacientes cada una. Al final del seguimiento, sólo $\mathrm{n}=402$ del total consiguieron la meta, $\mathrm{n}=10$ pacientes se perdieron en el seguimiento, $\mathrm{n}=12$ pacientes se excluyeron al tomar de manera equivocada la medicación y $n=$ seis pacientes no quisieron firmar el consentimiento.

Analizamos n = 214 en el grupo enoxaparina y $n=188$ en el grupo aspirina con un seguimiento a los 15, 30 y 90 días del postquirúrgico con más de $97 \%$ de apego a la profilaxis indicada.

Una parte importante de pacientes presentaba comorbilidades asociadas, otorgando una distribución de riesgo quirúrgico de 24, 69, 6 y 1\% para ASA I, II, III y IV respectivamente.

En total 402 rodillas operadas 221 derechas y $181 \mathrm{iz}$ quierdas con un tiempo promedio de estancia hospitalaria de 3.4 días y un promedio de pérdida sanguínea durante el procedimiento de $448 \mathrm{ml}$.

En cuanto al resultado primario, la eficacia de la tromboprofilaxis presentó un porcentaje de enfermedad tromboembólica de $1.24 \%(\mathrm{n}=$ cinco) en el total de la muestra, siendo $0.74 \%$ casos de TVP sintomática y $0.74 \%$ casos de EP, los cuales se confirmaron con USG, angio-TAC, ECO transesofágico. Se presentó un caso más de embolismo pulmonar (EP) en el grupo de enoxaparina sin ser significativo $(p=0.23)$.

El resultado de seguridad para tromboprofilaxis sin sangrado mayor en el total de la muestra. Sangrado menor de $1.74 \%$ ( $\mathrm{n}=$ siete), en el cual el hematoma en el sitio quirúrgico $1 \%$ ( $\mathrm{n}=$ cuatro) fue el de mayor incidencia, presentándose dos casos en ambos grupos; no hubo diferencias significativas $(p=0.82)$.

En referencia a resultados secundarios del análisis obtenido durante el seguimiento hubo $1.24 \%$ ( $\mathrm{n}=$ cinco) de infecciones del sitio quirúrgico.

En los primeros 15 días del procedimiento, $\mathrm{n}=$ dos en el grupo de enoxaparina y $\mathrm{n}=$ una en el de aspirina; pasados 30 días $\mathrm{n}=$ una infección de tejido superficial en cada grupo.

Una mujer de 80 años presentó un IAM a los 30 días del procedimiento y reingresó, por la misma causa, en el grupo de enoxaparina.

En cuanto al costo como variable aislada, el promedio por tratamiento por paciente con enoxaparina fue de 6,140.89 pesos mexicanos (MXN) aproximadamente vs. ácido acetilsalicílico (AAS), el cual tuvo un costo de alre- 
dedor de 72.50 MXN. Si realizamos la suma del gasto total en cada grupo, obtenemos un gasto total por población de enoxaparina de 1,252,742.36 pesos aproximadamente vs. cerca de 13,684.18 MXN de AAS.

\section{Discusión}

El estudio prospectivo aleatorizado demuestra que los pacientes que recibieron heparina de bajo peso molecular (enoxaparina) como tratamiento de tromboprofilaxis para una ATR electiva no es superior a la utilización de aspirina por 30 días con la dosis experimental.

La aspirina presentó $0.53 \%$ de EP en comparación con $0.93 \%$ en enoxaparina, sin ser significativo, además ambos presentaron un caso de TVP sintomática, por lo que se puede hablar de una efectividad similar.

Creemos que los hallazgos apoyan el uso de cualquiera de los fármacos, ya que la muestra contiene una distribución uniforme y es aplicable a la población en general. No se recomienda el uso de USG para diagnosticar TVP, ya que como se observó en el estudio, sólo se confirmaron pacientes sintomáticos quienes requirieron atención médica.

El uso de aspirina es una opción válida presente en la nuevas guías, aunque se pretende estudiar la dosis adecuada entre eficacia y seguridad; se cree que la dosis utilizada y el lapso terapéutico es el ideal para evitar complicaciones mayores por sangrados y no afectar la profilaxis, lo cual se mostró en los resultados con $1.59 \%(\mathrm{n}=$ tres $)$ de casos de sangrado menor con AAS vs. 1.89\% ( $n=$ cuatro) en el caso de enoxaparina, siendo el más frecuente en el total de la muestra el hematoma con $1.74 \%$. No se detectaron diferencias significativas entre grupos.
En cuanto a resultados secundarios, hubo $1.24 \%$ de complicaciones por infección, lo cual se correlaciona con la literatura actual para ATR, siendo el porcentaje total las infecciones superficiales.

En el grupo de enoxaparina se presentó un paciente a los 30 días con un IAM, el cual tuvo que reingresar al hospital. Se ha asociado a la aspirina con un efecto dual en otros estudios como WARFASA y ASPIRE protegiendo a los pacientes de eventos vasculares mayores y ofreciendo además protección para recurrencia de TVP; en el grupo de aspirina no hubo eventos vasculares mayores.

El costo por medida terapéutica es algo a tomar en cuenta, siendo que el promedio del tratamiento con enoxaparina representa 84 veces más el costo del tratamiento con aspirina.

La aspirina es una opción económica, por la que los sistemas de salud públicos y privados podrían optar para abaratar sus costos teniendo el mismo impacto; no requiere monitoreo, se puede utilizar en pacientes renales, además de no representar un riesgo de sangrado mayor durante el procedimiento y puede ser reversible con la transfusión plaquetaria. Es evidente que la administración es mucho más cómoda para el paciente y seguramente el apego al tratamiento es mejor, aunque en nuestra población no hubo diferencias en el mismo.

El estudio presenta limitaciones como el tamaño de la muestra, además de ser un estudio en un solo centro del país, el cual tuvo un escrutinio importante en la selección de pacientes para ATR electiva. Una de las mayores fortalezas fue el seguimiento, el cual se obtuvo en $100 \%$ de la muestra, además de un alto apego al tratamiento en ambos grupos.

Otra limitación es el escrutinio en criterios de inclusión, lo que limita la población analizada y excluye a pacientes

\begin{tabular}{|c|c|c|c|c|}
\hline Resultado & $\begin{array}{c}\text { Seguridad } \\
\mathrm{N}=402, \mathrm{n}(\%)\end{array}$ & $\begin{array}{c}\text { Enoxaparina } \\
\mathrm{N}=214, \mathrm{n}(\%)\end{array}$ & $\begin{array}{c}\text { Aspirina } \\
\mathrm{N}=188, \mathrm{n}(\%)\end{array}$ & $\mathrm{p}$ \\
\hline Sangrado mayor & & 0 & 0 & \\
\hline Sangrado menor & $7(1.74)$ & $4(1.86)$ & $3(1.59)$ & 0.823604 \\
\hline Hematoma & $4(1)$ & $2(0.93)$ & $2(1.06)$ & \\
\hline Gatrointesinal & 0 & 0 & 0 & \\
\hline Sangrado profuso del sitio quirúrgico & $1(0.25)$ & 0 & $1(0.53)$ & \\
\hline Equimosis & $2(0.50)$ & $2(0.93)$ & 0 & \\
\hline Hemoptisis & 0 & 0 & 0 & \\
\hline Hematuria & 0 & 0 & 0 & \\
\hline Hemorragia conjuntival & 0 & 0 & 0 & \\
\hline \multicolumn{5}{|l|}{ Resultados secundarios } \\
\hline Infección de la herida & $5(1.24)$ & & & 0.231768 \\
\hline Superficial & & $2(0.93)$ & $1(0.53)$ & \\
\hline Profunda & & 0 & 0 & \\
\hline Superficial $>30$ días & & $1(0.46)$ & $1(0.53)$ & \\
\hline Profunda $>30$ días & & 0 & 0 & \\
\hline IAM & & $1(0.46)$ & 0 & \\
\hline EVC & & 0 & 0 & \\
\hline Muerte & & 0 & 0 & \\
\hline
\end{tabular}


en condiciones en las que la dosis de aspirina podría ser modificada; aun así, pensamos que da una pauta bastante adecuada de la dosis y la duración de tratamiento ideal en un paciente electivo para ATR.

Se propone realizar estudios con poblaciones mayores y de manera multicéntrica con este antecedente con la finalidad de llegar a un consenso entre traumatólogos y elegir la mejor opción en nuestra práctica.

Se pueden llevar a cabo nuevos estudios con la misma dosis para pacientes sometidos a cirugía de cadera y columna electiva. Además es un punto de partida para el análisis de dosis en pacientes con factores de riesgo y/o comorbilidades que afectan la coagulación.

\section{Conclusiones}

Se puede concluir que la aspirina y la enoxaparina son agentes seguros y eficaces sin diferencias significativas en la población que se somete a una ATR electiva, por lo que debería formar parte del cuadro terapéutico y ser opción para el paciente.

Se considera que el esquema experimental de aspirina es el ideal, ya que se cuenta con esa posología en el mercado. Es una opción económica y cómoda para el paciente.

Referencias

1. Azboy I, Barrack R, Thomas AM, Haddad FS, Parvizi J. Aspirin and the prevention of venous thromboembolism following total joint arthroplasty: commonly asked questions. Bone Joint J. 2017; 99B(11): 1420-30. doi: 10.1302/0301-620X.99B11.BJJ-2017-0337.R2.

2. Lussana F, Squizzato A, Permunian ET, Cattaneo M. A systematic review on the effect of aspirin in the prevention of post-operative arterial thrombosis in patients undergoing total hip and total knee arthroplasty. Thrombosis Research. 2014; 134(3): 599-603. doi: 10.1016/j.thromres.2014.06.027.

3. Behan MW, Storey RF. Antiplatelet therapy in cardiovascular disease. Postgrad Med J. 2004; 80(941): 155-64. doi: 10.1136/ pgmj.2003.007062.

4. Awtry EH, Loscalzo J. Aspirin. Circulation. 2000; 101(10): 1206-18. doi: 10.1161/01.cir.101.10.1206.
5. Jenny JY, Pabinger I, Samama CM; ESA VTE Guidelines Task Force. European guidelines on perioperative venous thromboembolism prophylaxis: Aspirin. Eur J Anaesthesiol. 2018; 35(2): 123-129. doi: 10.1097/EJA.0000000000000728.

6. An VV, Phan K, Levy YD, Bruce WJ. Aspirin as thromboprophylaxis in hip and knee arthroplasty: a systematic review and meta-analysis. $J$ Arthroplasty. 2016; 31(11): 2608-16. doi: 10.1016/j.arth.2016.04.004.

7. Lieberman JR, Heckmann N. Venous thromboembolism prophylaxis in total hip arthroplasty and total knee arthroplasty patients: from guidelines to practice. J Am Acad Orthop Surg. 2017; 25(12): 789-98. doi: 10.5435/JAAOS-D-15-00760.

8. Gutowski CJ, Zmistowski BM, Lonner JH, Purtill JJ, Parvizi J. Direct costs of aspirin versus warfarin for venous thromboembolism prophylaxis after total knee or hip arthroplasty. J Arthroplasty. 2015; 30(9 Suppl): 36-8. doi: 10.1016/j.arth.2015.04.048.

9. Anderson DR, Dunbar MJ, Bohm ER, Belzile E, Kahn SR, Zukor $\mathrm{D}$, et al. Aspirin versus low-molecular-weight heparin for extended venous thromboembolism prophylaxis after total hip arthroplasty: a randomized trial. Ann Intern Med. 2013; 158(11): 800-6. doi: 10.7326/0003-4819-158-11-201306040-00004.

10. Lieberman J, Berry D, Azar F. Advanced reconstruction knee. Rosemont, IL: American Academy of Orthopaedic Surgeons (AAOS); 2011.

11. Azboy I, Groff H, Goswami K, Vahedian M, Parvizi J. Low-dose aspirin is adequate for venous thromboembolism prevention following total joint arthroplasty: a systematic review. J Arthroplasty. 2020; 35(3): 886-92. doi: 10.1016/j.arth.2019.09.043.

12. Mostafavi Tabatabaee R, Rasouli MR, Maltenfort MG, Parvizi J. Costeffective prophylaxis against venous thromboembolism after total joint arthroplasty: warfarin versus aspirin. J Arthroplasty. 2015; 30(2): 159-64. doi: 10.1016/j.arth.2014.08.018.

13. Harenberg J, Jorg I, Fenyvesi T. Heparin-induced thrombocytopenia: pathophysiology and new treatment options. Pathophysiol Haemost Thromb. 2002; 32(5-6): 289-94. doi: 10.1159/000073583.

14. Warkentin TE. Aspirin for dual prevention of venous and arterial thrombosis. N Engl J Med. 2012; 367(21): 2039-41. doi: 10.1056/NEJMe1211480.

15. Wendelboe AM, St Germain L, Krolak B, Reiser T, Raskob G; ISTH Steering Committee on World Thrombosis Day. Impact of world thrombosis day campaign. Res Pract Thromb Haemost. 2017; 1(1): 138-41.

16. Wolberg AS, Rosendaal FR, Weitz JI, Jaffer IH, Agnelli G, Baglin T, et al. Venous thrombosis. Nat Rev Dis Primers. 2015; 1: 15006.

17. Bang SM, Jang MJ, Kim KH, Yhim HY, Kim YK, Nam SH, et al. Prevention of venous thromboembolism, 2nd edition: Korean Society of Thrombosis and Hemostasis Evidence-based Clinical Practice Guidelines. J Korean Med Sci. 2014; 29(2): 164-71.

18. Buesing KL, Mullapudi B, Flowers KA. Deep venous thrombosis and venous thromboembolism prophylaxis. Surg Clin North Am. 2015; 95(2): 285-300. 